\title{
Correction to: AIDS Patient Care STDs 2016;30:247-253; DOI: 10.1089/apc.2016.0068
}

\begin{abstract}
In the article, "Identifying Areas for Improvement in the Screening Process of a HighPrevalence Emergency Department," published in AIDS Patient Care and STDs 2016;30:247-253, the senior author is listed as Sobha Swaminthan, but her name was misspelled. The correct spelling is Sobha Swaminathan. The online version of the article has been corrected to reflect this change.
\end{abstract}

The authors regret this error. 